\title{
Functional Popliteal Artery Entrapment Syndrome: An Approach to Diagnosis and Management
}

\author{
Authors \\ Thomas Lovelock, Matthew Claydon, Anastasia Dean
}

\author{
Affiliation \\ Department of Vascular Surgery, Alfred Hospital, \\ Melbourne, Australia
}

Key words

functional PAES, chronic exertional compartment syndrome, exertional leg pain

accepted 27.05.2021

published online $\quad 02.08 .2021$

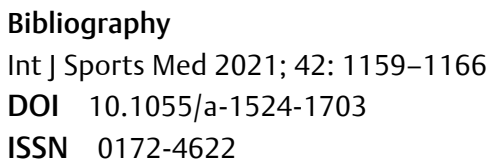

丹upplementary Material is available under https://doi.org/10.1055/a-1524-1703.

\begin{abstract}
Popliteal Artery Entrapment Syndrome (PAES) is an uncommon syndrome that predominantly affects young athletes. Functional PAES is a subtype of PAES without anatomic entrapment of the popliteal artery. Patients with functional PAES tend to be younger and more active than typical PAES patients. A number of differential diagnoses exist, the most common of which is chronic exertional compartment syndrome. There is no consensus regarding choice of investigation for these patients. However, exercise ankle-brachial indices and magnetic resonance imaging are less invasive alternatives to digital subtraction angiography. Patients with typical symptoms that are severe and repetitive should be considered for intervention. Surgical intervention consists of release of the popliteal artery, either via a posterior or medial approach. The Turnipseed procedure involves a medial approach with a concomitant release of the medial gastrocnemius and soleal fascia, the medial tibial attachments of the soleus and excision of the proximal third of the plantaris muscle. Injection of botulinum A toxin under electromyographic guidance has recently shown promise as a diagnostic and/or therapeutic intervention in small case series. This review provides relevant information for the clinician investigating and managing patients with functional PAES.
\end{abstract}

\section{Introduction}

Popliteal artery entrapment syndrome (PAES) was first described by Anderson Stuart, a medical student at the University of Edinburgh in 1879, who while dissecting out a cadaver with a large popliteal aneurysm noted an aberrant course of the popliteal artery medial to the medial head of gastrocnemius (MHOG).

PAES is an uncommon syndrome that typically affects young athletes, with a strong predilection for males [1]. The estimated prevalence is somewhere between $0.17-3.5 \%$ [2, 3]. Traditionally, PAES has been defined as an entrapment syndrome secondary to an abnormal relationship between the popliteal artery and the adjacent muscle and tendons [4]. This may be as a consequence of an anomalous route of the popliteal artery, abnormality in the development or insertion of the MHOG, or compression of the artery by an accessory ligament or fibrous band $[4,5]$. This entrapment may cause intermittent claudication in younger patients typically without atherosclerotic risk factors [2,6]. Approximately 30 percent of patients have bilateral PAES [7].

\section{Materials and Methods}

\section{Literature Review}

A search of PubMed, Scopus and the Cochrane Collaboration was performed using a combination of keywords including truncated and wildcard variations and relevant subject headings, focusing on PAES and functional PAES, as well as terms relevant to the succeeding subheadings. Non-English abstracts were not included. Reference lists of reviewed articles were trawled to identify relevant articles not identified in the literature search. 


\section{Anatomy of the Popliteal Artery and Classification of PAES}

The popliteal artery passes from the adductor hiatus into the popliteal fossa, where it typically passes lateral to the medial head of the gastrocnemius, and runs down posterior to the knee joint, superficial to the popliteus muscle. At the lower border of the popliteus, it divides into the anterior tibial artery and tibioperoneal trunk [8]. The popliteal artery is fixed at both ends, to the adductor hiatus and to the soleal fascia [8,9]. It is worth noting that over 75 variations of this "normal" anatomy have been described [10], which may impact on the presentation and pathogenesis of PAES.

A classification system for PAES was proposed by Love and Whelan and further modified by Rich [11], who described six subtypes of PAES, defined by the relationship of the popliteal artery to the medial head of gastrocnemius (MHOG) ( Figure 1a-f). Type 1 PAES describes a variation whereby the popliteal artery runs medial to the MHOG. This occurs because the popliteal artery matures prior to the medial migration of the MHOG and is swept medially by the migrating muscle. In type 2, the popliteal artery is again medial to the MHOG, but it partially arrests the medial migration of the MHOG, such that the muscle attaches more laterally on the femur. In type 3, an accessory band of gastrocnemius entraps the popliteal artery. Part of the axial popliteal artery does not involute in type 4, so the mature distal popliteal artery runs deep to the popliteus muscle. If the popliteal vein accompanies the popliteal artery in any of these anatomical variations, it is termed type 5 . In type 6 , none of the above anatomical variations are present $[2,11]$. Type 6 is also known as functional popliteal entrapment syndrome (Functional PAES).

Functional PAES defines a group of patients who experience reproducible symptoms of PAES without an identifiable developmental anatomical abnormality [10]. Rignault described this in 1985, having studied in digital subtraction angiography (DSA) of French military recruits' lateral displacement of the popliteal artery at rest, and occlusion of the artery with plantarflexion. At operation, no anatomical cause was found in these patients, aside from hypertrophy of the medial gastrocnemius muscle belly [2,12].

The exact mechanism of functional PAES is incompletely understood. As aforementioned, the pathology was initially believed to occur secondary to hypertrophy of the medial gastrocnemius muscle in athletes, with compression of the posteromedial aspect of the popliteal artery during stress maneuvers $[12,13]$. Others have postulated that the neurovascular bundle - the popliteal artery, popliteal vein, and tibial nerve - is compressed where it passes below the soleal arch (the so-called "popliteal outlet"), the tendinous band that spans between the muscle's proximal attachment at the tibia and the head of the fibula, and that symptoms may arise from compression of the tibial nerve at this point. Hypertrophy of the soleus, gastrocnemius, plantaris and popliteus muscles may contribute to this compression [12-14]. One study using Magnetic Resonance Imaging (MRI) found that the insertion of the medial head of gastrocnemius may extend further towards the midline of the femur, further crowding the region [15].

\section{Clinical Features of Functional PAES}

Functional PAES predominantly affects young, fit individuals and presents with symptoms of exertional calf pain, weakness, cramp- ing and a sensation of tenseness. There may be associated paraesthesia of either the dorsal or plantar aspect of the foot $[1,16]$. It is unclear whether the genesis of these symptoms is from ischemic pain secondary to popliteal artery occlusion or compression of the neurovascular bundle (including the tibial nerve) as it passes under the soleal arch [14]. Compression of the tibial nerve (and its innervation of the dorsum of the foot via its calcaneal and plantar branches) may explain the frequent complaint of paraesthesia of the plantar surface of the foot. Compared to anatomical (type 1 to 5) PAES, patients with functional PAES tend to be younger, more active, and more commonly female $[3,17]$. There is often a significant delay between the onset of symptoms and diagnosis, owing to their non-specific nature $[18,19]$.

There are no specific examination signs indicative of functional PAES. Patients may present with hypertrophy of the lower limbs [4]. There may be a loss of the dorsalis pedis or posterior tibial pulse on resisted plantar or dorsiflexion with the knees extended [20,21]. A drop in the Ankle-Brachial Index ( $\mathrm{ABI}$ ) or significant flattening of a plethysmography waveform with these same maneuvers is considered a positive "entrapment test" [16]. A positive entrapment test and symptoms of atypical claudication are not of themselves sufficient to make the diagnosis of functional PAES, as somewhere between $20-85 \%$ of normal legs may demonstrate occlusion of the popliteal artery on duplex ultrasound or MRI in plantarflexion $[10,22]$. Provocative maneuvers, such as hopping on one leg, can induce symptoms including weakness and paraesthesia of the toes; and the foot can appear cold and pale, followed by reactive hyperemia following cessation of exercise [23].

Potential long-term sequelae of anatomical PAES stem from the effects of repetitive arterial micro-trauma on the popliteal artery and include distal embolization, dissection, acute limb ischemia secondary to acute thrombosis, post-stenotic dilatation and aneurysm formation $[4,7,21]$. This repetitive micro-trauma can be divided into three phases: in the first phase there is damage to the arterial adventitia; in the second, damage to the media; and in the third, the intima. Damage to the media may cause post-stenotic dilatation or aneurysmal degeneration of the artery, while damage to the intima can lead to thrombosis [24]. Sinha's 2012 meta-analysis reported a median prevalence of $24 \%$ of popliteal artery occlusion and $13.5 \%$ of post-stenotic dilatation or aneurysm formation amongst patients with PAES [7]. There are reports of patients presenting with complications from functional PAES; however, the incidence of these complications appears to be much rarer than in their anatomical counterparts [24].

\section{Differential Diagnosis}

Functional PAES represents one of a number of diagnoses that exist on the spectrum of exercise induced leg pain. These differential diagnoses are important to consider, as many may masquerade as functional PAES.

\section{Vascular causes of exertional leg pain}

Arterial endofibrosis is a non-atherosclerotic cause of exertional leg pain in young athletes, particularly cyclists [25]. It primarily afflicts the external iliac artery [26]. The exact pathophysiology of the disease is unknown but there is suspected to be an association between duration of time spent cycling and the development of 

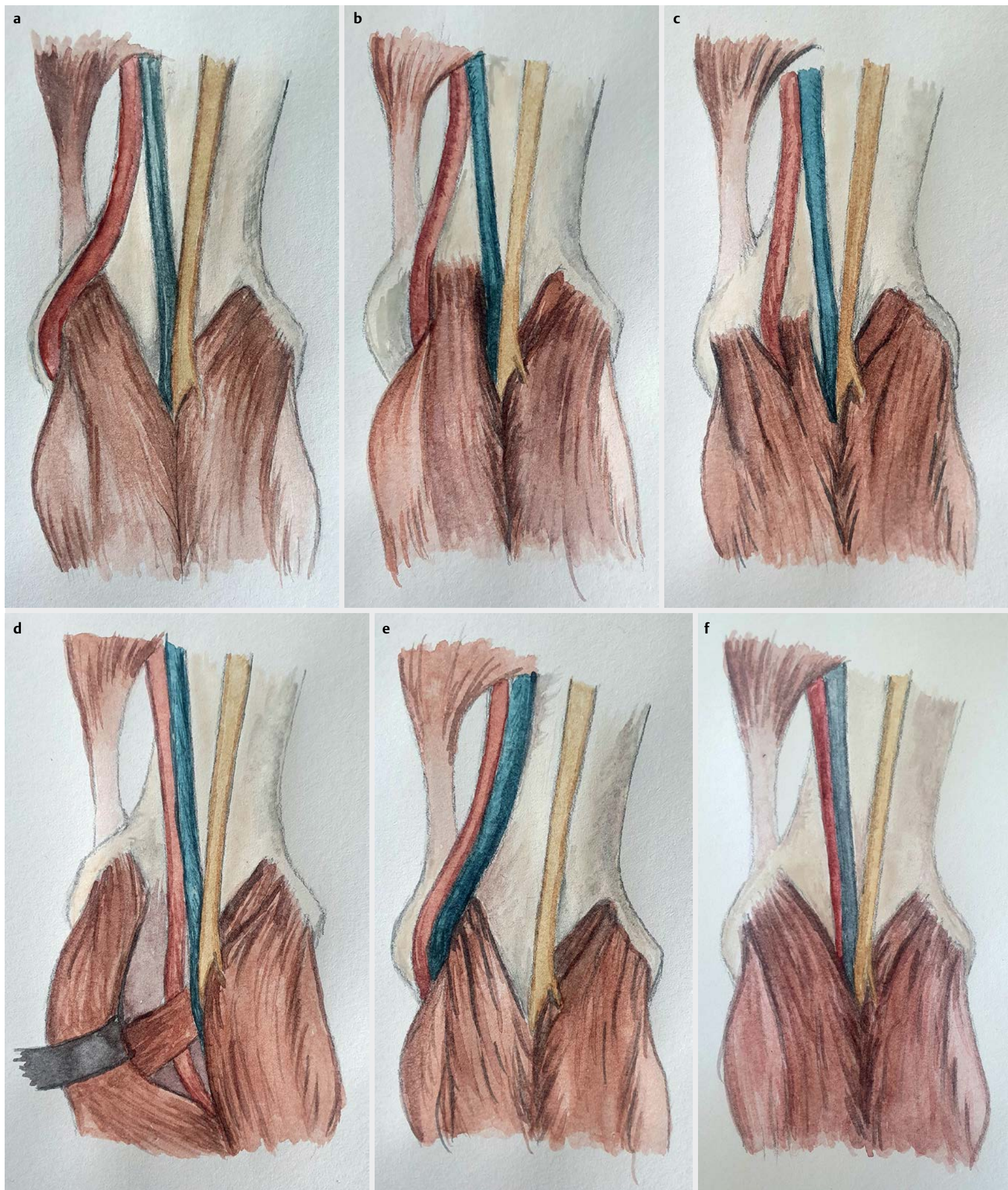

- Fig. 1 Subtypes of Popliteal Artery Entrapment Syndrome (PAES). In Type 1, the popliteal artery runs medial to the medial head of gastrocnemius (MHOG) a. In Type 2, the popliteal artery again runs medial to the MHOG; however, the MHOG has a more lateral attachment on the femur $\mathbf{b}$. In Type 3 , an accessory band of gastrocnemius entraps the popliteal artery $\mathbf{c}$. In Type 4, the popliteal artery runs deep to the popliteus muscle $\mathbf{d}$. If the popliteal vein accompanies the artery in any subtype it is classified as Type 5 e. In Type 6 , functional PAES, there is no anatomical abnormality present $\mathbf{f}$. 
the disease [25, 26]. Typical symptoms include leg weakness and thigh pain on exertion, which resolve with cessation of exercise. Patients will have a drop in exercise ankle-brachial index pressures, of at least $20-40 \%$ [25]. Duplex ultrasonography may demonstrate increased intimal-medial thickness, and exercise duplex ultrasonography may show elevated peak systolic velocities (>350 cm/s) with dampened waveforms [20]. DSA may show iliac artery stenosis or occlusion, with kinking of the vessels either at rest or when provoked with hip flexion. Most commonly, this is managed with open endarterectomy and patch angioplasty [20], while surgical release of the iliac artery from its sheath to prevent kinking has also been described [27]. Endovascular interventions may offer temporary relief; however, they have not been shown to confer long-term results [20].

Adductor canal compression syndrome is a rare entrapment condition where the superficial femoral artery (SFA) is compressed in the adductor canal. Etiology can be either anatomical, with compression of the SFA by fibrous or musculotendinous bands arising from the adductor magnus, or functional, where the SFA is compressed between the hypertrophied vastus medialis and adductor magnus muscles [28]. Patients either present with intermittent claudication or medial knee pain secondary to compression of the saphenous nerve [29]. Relevant investigations include exercise ABIs, duplex ultrasonography, Computed Tomography (CT) angiography, and MRI [28]. Management of symptomatic individuals is with open surgery, namely patch angioplasty or bypass of the affected region, accompanied by removal of any accessory musculotendinous or fibrous bands [28].

Cystic adventitial disease is a rare condition of mucinous cyst formation within the adventitia of the artery [30]. This disease affects males between 5 to 15 times more than females, and occurs approximately $85 \%$ of the time in the popliteal artery [31]. Patients typically experience short distance claudication, with a prolonged recovery of symptoms after cessation of exercise [32]. Ishikawa's sign (loss of pedal pulses with knee flexion) may be present [31]. Duplex ultrasonography is the most sensitive test for the disorder, although CT angiography and MRI may also prove useful [31]. DSA demonstrates a smooth curvi-linear stenosis, referred to as the "scimitar sign" [30, 31]. In the symptomatic patient, treatment options include resectional (resection of diseased vessel with patch or interposition graft repair) and non-resectional techniques (such as ultrasound guided cyst aspiration) $[30,31]$. Non-resectional techniques have a higher rate of recurrence and often a need for repeat intervention [31]. Endovascular approaches have not been shown to be successful in this setting [30].

\section{Non-vascular causes of exertional leg pain}

Chronic Exertional Compartment Syndrome (CECS) is the most common cause of atypical claudication in the young athlete, occurring in approximately $30 \%$ of athletes with exertional leg pain $[16,33]$. It may present alongside PAES, with one cohort of patients with PAES reported to have an $86 \%$ incidence of concomitant CECS [20], and is a mandatory consideration in the work-up of exertional leg pain. Many functional PAES patients may have already undergone surgery for CECS $[23,34]$. It is predominantly reported in young athletes who run, but may also affect non-athletes [35]. Symptoms include pain over one of the four leg compartments, which increases during exercise, and resolves within minutes to hours of ceasing activity [35]. Generally, there are no examination findings, although there may be point tenderness or muscle atrophy in the affected compartment [35]. The investigation of choice is intra-compartmental pressures, measured at rest, and both 1 and 5 minutes after exercise $[35,36]$. More recently, the reproducibility of results between different intra-compartmental pressure measuring systems, and the accuracy of proposed diagnostic criteria have been brought into question [35]. Accordingly, the diagnosis remains largely a clinical one. Non-operative strategies including massage, gait changes and chemo-denervation appear to be successful in approximately 50 percent of cases [36, 37]. Operative management includes fasciotomy, which can be undertaken either via a single (lateral) or double (anterolateral and medial) incisions [36]. It is important to ensure that all compartments, including the deep posterior compartment, are decompressed. Alternative techniques include endoscopically assisted fasciotomy and minimally invasive fasciotomy, which appear to confer similar results [35, 36]. Given the similarities in presentation between functional PAES and CECS, patients may often present with overlapping symptoms. Where there is uncertainty as to which is the predominant cause of the patient's symptoms, it has been advocated to perform fasciotomies first $[16,19]$.

Compression of the tibial nerve as it passes under the soleal sling ("Soleal Sling Syndrome") is gaining recognition as a separate entity to functional PAES and CECS. In this syndrome, patients develop plantar numbness or pain, with associated pain or tightness in the calf [38]. As there is no popliteal artery compression, patients do not experience the claudication symptoms typical of functional PAES [38]. As previously mentioned, there is likely a degree of overlap between patients with functional PAES and soleal sling syndrome, as patients with functional PAES often experience symptoms of plantar pain or numbness. Patients often have their plantar numbness mistaken for tarsal tunnel syndrome, and may have already undergone unsuccessful surgical decompression of this $[38,39]$. A key examination finding is tenderness on palpation of the tibial nerve as it passes under the soleal sling (approximately $9 \mathrm{~cm}$ below the posterior knee crease) $[38,39]$. Patients may have sensory changes in the distribution of the tibial nerve, and there may be weakness, particularly in extensor hallucis longus [39]. MRI may demonstrate a thickened soleus sling with $\mathrm{T} 2$ hyperintensity of the compressed tibial nerve $[40,41]$. Conservative management involves modifying activities to avoid those that induce pain and wearing non-constrictive clothing and shoes [39]. Surgical decompression involves detaching the soleal arch, via a medial calf incision, with promising results from the small case numbers reported thus far [38, 39].

Medial tibial stress syndrome (MTSS) and stress fractures are two other important differential diagnoses to consider in the young patient with exertional leg pain. MTSS is a common affliction in runners with symptoms of pain at the posteromedial border of the tibia after exercise. Symptoms typically persist for hours to days (compared with minutes to hours of CECS) [36, 42]. Patients with stress fractures, however, experience pain at a focal site, which worsens over time as training loads increase. They may also experience significant pain at night [35]. Patients with MTSS will experience diffuse pain across the medial aspect of the tibia, while patients with 
stress fractures will have tenderness concentrated over an area of less than $5 \mathrm{~cm}$ [35]. Plain X-ray imaging is a primary method of investigation, and MRI is the preferred choice [35]. Both conditions should be managed conservatively [35].

Plantar fasciitis is a degenerative condition, with overuse or excessive loading causing repeated micro-trauma to the plantar fascia. It typically presents with localized heel pain, though in severe cases, the pain can radiate proximally into the lower limb $[43,44]$. The pain is typically worst early in the morning or after prolonged activity. Risk factors include anatomical deformity (pes planus and pes cavus) and obesity, and it is more prevalent in athletes and the elderly. On examination, focal tenderness at the origin of the plantar fascia is expected. Pain may also be elicited with passive dorsiflexion. Some patients with plantar fasciitis have limited ankle dorsiflexion, attributable to gastrocnemius tightness [43, 44]. In plantar fasciitis, there is not any reduction in sensitivity in the innervation of the plantar nerves, in contrast to tarsal tunnel syndrome where this is commonplace. Recently, surgical resection of the medial head of gastrocnemius (a typical surgical treatment for functional PAES) and gastrocnemius tendon lengthening have been investigated as alternatives to traditional open plantar fasciotomy (albeit in small numbers) $[43,44]$.

Lower back pain may be accompanied by leg pain. This may either be referred pain, where pathology in the muscles, joints or ligaments in the lumbar spine or pelvis (including the sacroiliac joint) may produce both pain at the site of nociception and also in the leg, or radicular pain, where there is shooting pain down the leg as a result of lumbar intervertebral disc herniation [44-47]. Both of these syndromes can have a gradual or sudden onset. Referred pain is provoked by mechanical pressure on the afflicted structures and tends to be more dull or aching, while radicular pain is elicited by stretching the neural elements through standing or bending and is described as shocking and electric in character. Both can be severe and debilitating for patients. History and examination, including straight leg raise and femoral stretch tests, may help delineate the cause. Patients with radicular pain may have motor, sensory or reflexive deficits in their limbs. Imaging, including plain $\mathrm{X}$-ray and MRI scans, nerve conduction studies and electromyography, may be indicated [44-47]. Treatment of referred pain is directed towards increasing mobility and return to pre-morbid function levels, whereas radicular pain treatment ranges on a spectrum from conservative management with analgesia and physiotherapy to surgery [47].

\section{Investigations}

There is no consensus as to the best series of investigations for functional PAES. Investigations most commonly performed include exercise ankle-brachial pressures (exercise ABIs) and duplex ultrasound (typically performed with the ankle in dorsiflexion and plantarflexion), CT angiography, MRI (encompassing plain MRI, stress positional MRI and MR angiography), and DSA [7, 48].

Most patients with anatomical and functional PAES will have normal resting $A B I s$ [49]. Turnipseed and others have suggested that exercise $A B I s$ should be the first investigation of choice when diagnosing functional PAES [3]. Exercise ABIs have been shown to successfully distinguish symptomatic from asymptomatic patients in a cohort of young patients with exertional leg pain, with the greatest difference demonstrated after strenuous exercise, specifically running [50]. ABIs should be measured after exercise vigorous enough to produce symptoms (such as running outside or at high speed on a treadmill) to maximize sensitivity. Furthermore, the test should also be performed as quickly as possible after cessation of exercise as pressures may return to baseline rapidly in these patients [23].

Duplex ultrasound with dynamic maneuvers (ankle dorsi and plantar-flexion) is often the first test ordered when specifically investigating for functional PAES. There are no defined ultrasound criteria for the diagnosis of popliteal entrapment [24], and between $20-85 \%$ of normal legs may demonstrate transient occlusion of the popliteal artery on duplex ultrasound with dynamic maneuvers, leading to a high rate of false positives [10]. Typically, the popliteal artery is examined in the region where it is thought to be compressed by the medial head of gastrocnemius; however, this does not investigate for compression of the neurovascular bundle at the "popliteal outlet," a prominent etiological theory in functional PAES. One study examining compression of the popliteal artery at this "popliteal outlet" found that only $10 \%$ of a cohort of young patients with exertional leg pain had popliteal artery occlusion when examined with dynamic ultrasound with ankle plantarflexion [50], with mean compression of the artery that was less than $2.5 \mathrm{~mm}$ [50]. This technique has not, however, been validated in a specific cohort of patients with suspected functional PAES.

CT angiography (CTA) using iodinated contrast media is a less invasive imaging modality that provides both high spatial resolution and good soft tissue contrast resolution [51]. It may prove a useful investigation in the work-up of patients for atypical causes of claudication. Separately phased scans can be performed with ankle dorsiflexion or plantarflexion to investigate specifically for evidence of popliteal entrapment [51]. Plethysmography using an oximeter on the hallux of the afflicted side can ensure the ankle has been sufficiently positioned or flexed $[16,52]$. CTA may demonstrate mild to severe popliteal artery stenosis, popliteal artery occlusion, and even popliteal venous compression [51]. Multiplanar reconstructions and maximal intensity projections allow good visualization of the complete course of the artery and may identify muscular or tendinous abnormalities in the case of anatomical PAES [53]. Alternative diagnoses such as cystic adventitial disease may also be readily identified [53]. Its less invasive nature has led many to prefer it over DSA in the investigation of functional PAES [53]. Some patients with symptoms of PAES and signs of popliteal artery compression on CTA demonstrate minimal drop in pressure on exercise $A B l s$ [52] - it is unclear whether this subset of patients did not exercise vigorously enough to incur a drop in $A B I$, or if there is a subset of patients with functional PAES who have symptoms and imaging consistent with the diagnosis, but normal exercise ABIs.

MRI is a valuable imaging modality to assess the lower limb arterial tree and soft tissue structures. It is the most commonly used investigation in the work-up for functional PAES [48]. MRI has greater specificity and sensitivity in the detection of lower limb peripheral arterial disease, when compared to duplex ultrasound [54] and does not expose the patient to ionizing radiation, an important consideration in this typically younger cohort of patients. T1weighted MRI sequences display the muscular anatomy and its relation to the popliteal artery, and as such can be used to diagnose 
or exclude anatomical PAES as the cause of the patient's symptoms $[55,56]$. Time-of-flight MR angiography (MRA) can demonstrate arterial compression in either ankle dorsiflexion or plantarflexion without the need for administration of intravenous contrast media. However, MRI is acutely sensitive to motion artefacts, and the scan duration is typically longer than CTA [55]. Contrast-enhanced MRA may act as a less invasive substitute for DSA, with correlation between MRI and DSA findings of approximately $95 \%$ in patients with lower limb atherosclerotic disease, although MRI may tend to underestimate the degree of stenosis [57]. Use of blood pool MRI contrast agents, which stay in the intravascular space for a longer period of time, may allow for the acquisition of higher spatial resolution sequences, which may overcome the tendency to underestimate the degree of stenosis, as well as permitting arterial imaging with dynamic maneuvers [58]. There are a number of absolute and relative contraindications to MRI, including some implantable cardiac devices, endovascular stent grafts and orthopedic implants to name a few. These may prohibit the use of MRI, and hamper image quality if they are within the region of interest. Similarly, gadolinium-based contrast is contraindicated in patients with severe kidney impairment due to the risk of nephrogenic systemic fibrosis. These contraindications are less commonly encountered in the typically young, fit cohort of functional PAES patients.

DSA is still considered by many to be the "gold standard" imaging modality in the identification of patients with functional PAES [51]. DSA with stress maneuvers may identify a stenosis or occlusion of the popliteal artery if present, either at rest or with ankle dorsi- and plantarflexion [51, 59]; however, it is an invasive procedure and it exposes the patient to ionizing radiation. DSA can be particularly useful when MRA is inconclusive, or the diagnosis is still suspected despite normal MRA findings. In patients with complicated presentations, DSA could allow for simultaneous therapeutic endovascular intervention in the rare situation of a patient presenting with acute limb ischemia [60].

\section{Management}

In patients with anatomical PAES, surgery is indicated to release the entrapment and prevent complications such as arterial stenosis, occlusion and aneurysm formation. It appears that functional PAES is much less likely to result in these complications. Thus, intervention is generally reserved for patients with functional PAES who have typical symptoms that are severe and repetitive $[12,17]$. Non-operative intervention is in the form of massage and stretching with the intent to reduce gastrocnemius contraction and retraction, although this is unlikely to be effective as it does not affect muscle morphotype or volume [61].

Botulinum Toxin A (BTA) has emerged in the past five years as a non-operative treatment for functional PAES. BTA is injected under electromyographic guidance into the medial head of gastrocnemius and/or the plantaris muscles [48]. Typically, between one and three treatments are performed, with the further "top up" treatments provided at patient request $[48,62]$. In total, less than 50 patients cumulatively have been reported in the literature as having undergone this technique $[48,61,62]$. Post-procedural success rates appear to be greater than 50 percent, and advantages of this therapy are that it is minimally invasive and potentially diagnostic in identifying patients who are likely to benefit from surgery. It may function as a bridge to surgery, and in particular cases, alleviate the need for surgery altogether [48]. The long-term outcomes of BTA therapy for functional PAES remain unclear. It is less likely to be effective, if there is already arterial pathology, such as popliteal artery stenosis or occlusion [48].

Surgery remains the most widely accepted treatment for functional PAES. Three predominant surgical approaches have been described: posterior, medial and the Turnipseed approach. A posterior approach may be undertaken by a lazy S incision over the popliteal fossa. The popliteal fascia is incised, protecting the sural nerve and short saphenous vein, to expose the medial head of gastrocnemius. This is divided using either a right angle and diathermy, or a LigaSure [23,52]. The plantaris may also be divided at this point [63]. Proponents of this approach state that it allows better access and visualization of the neurovascular bundle, and easy access to the medial head of gastrocnemius [13, 16, 23, 52]. Potential drawbacks are the difficulty of proximal arterial access, should an arterial bypass be necessary, and that the patient must be positioned in a prone position. The medial approach is via an incision in the medial lower thigh, facilitating access to the above-knee popliteal artery. This allows division of the medial head of gastrocnemius, but does not permit division of the plantaris or popliteus [34]. Advantages of this approach are that patients are supine for the procedure, and that more proximal access to the artery may be gained if a bypass is required [24]; additionally, it provides easy access to the greater saphenous vein. However, visualization of the distal popliteal artery, particularly at the level of the "popliteal outlet," is more difficult. The final approach is that described by Turnipseed. A superomedial calf incision is made, similar to that for exposure of the below-knee popliteal artery. The fascial attachments of the gastrocnemius and soleus are excised off the medial tibia. The tendon and proximal third of the plantaris muscle are excised. The soleus is taken down at its medial attachment to the tibia. The anterior soleal fascia is taken down at the level of the popliteal outlet [16]. Possible advantages of this procedure are that it spares resection of the musculature of the medial head of gastrocnemius (which may be less debilitating in an athletic cohort of patients), and that it releases the medial tibial origin of the musculature and the anterior soleal fascia which may cause compression of the neurovascular bundle at the popliteal outlet. Concerns, however, have been raised that insufficient debulking of the medial head of gastrocnemius may result in post-operative compression of the popliteal vein, even if the artery has been successfully freed [23,52]. It is unclear whether this is a possibility in patients undergoing this technique. Another possible drawback of the approach is the limited access to the above-knee popliteal artery. Currently, there is no comparative data regarding the relative efficacy of these approaches.

Vascular reconstruction has not been reported in patients with functional PAES [48]. Experience extrapolated from patients with anatomical PAES suggests that treatment would involve thromboendarterectomy with or without patching or interposition or exclusion bypass, as vessel damage generally necessitates resection of the intima and/or media [24, 48]. Data from patient cohorts who have undergone vascular reconstruction for anatomical PAES suggests that the 5- and 10-year patency rates of these reconstructions are excellent (>90\%); however, patients who require exten- 
sive or distal bypass have a significantly lower patency rate, approaching $20 \%$ in Lejay's cohort $[5,64,65]$.

There is no validated method of assessing post-operative outcomes in functional PAES. Some authors have measured the percentage of patients who have successfully returned to their premorbid level of sport post-operatively [52], while others have recorded post-procedural exercise ABIs and compared them to pre-procedural figures [23]. There is also no evidence regarding whether patients should be surveilled long-term after surgery. Qualitative observations suggest that long-term recurrence in patients with functional PAES may be secondary to fibrosis around the neurovascular bundle [23].

Endovascular treatment of functional PAES has not been described. Endovascular treatment is not recommended for the management of anatomical PAES. An endovascular approach is not preferred in functional PAES, as it does not correct the compression of the popliteal artery. Furthermore, there are concerns regarding the durability of endovascular angioplasty and stenting in young patients.

\section{Conclusion}

This review provides relevant information for the clinician investigating and managing patients with functional PAES. This is an uncommon cause of claudication in younger patients, and can often prove difficult to diagnose. A number of investigations for the disorder exist, without a clear investigation of choice. Patients with significant and repetitive symptoms of functional PAES should undergo treatment, with surgical management forming the mainstay. Future frontiers in the investigation and management of functional PAES include development of specific imaging pathways and guidelines, the emergence of Botulinum Toxin A as a treatment or therapeutic investigation, and validation of outcome measures to quantify and monitor improvement in patient symptoms after management.

\section{Conflict of Interest}

The authors state that this study meets the ethical standards of the journal [66]. The authors have no conflicts of interest to declare.

\section{References}

[1] Wady H, Badar Z, Farooq Z et al. Avoiding the trap of misdiagnosis: Valuable teaching points derived from a case of longstanding popliteal artery entrapment syndrome. Case Rep Med 2018; 2018: 3214561

[2] Grimm N, Danilkowicz R, Shortell C et al. Popliteal artery entrapment syndrome. JBJS Rev 2020; 8: e0035

[3] Turnipseed W. Popliteal entrapment in runners. Clin Sports Med 2012; 31: $321-328$

[4] Gokkus K, Sagtas E, Bakalim T et al. Popliteal entrapment syndrome. A systematic review of the literature and case presentation. Muscles Ligaments Tendons J 2014; 4: 141-148

[5] Igari K, Sugano N, Kudo T et al. Surgical treatment for popliteal artery entrapment syndrome. Ann Vasc Dis 2014; 7: 28-33
[6] Hicks C, Black ], Ratchford E. Popliteal artery entrapment syndrome. Vasc Med 2019; 24: 190-194

[7] Sinha S, Houghton P, Holt et al. Popliteal entrapment syndrome. J Vasc Surg 2012; 55: 252-262

[8] Aktan Ikiz Z, Ucerler H, Ozgur Z. Anatomic variations of popliteal artery that may be a reason for entrapment. Surg Radiol Anat 2009; 31: 695-700

[9] Ozgur Z, Ucerler H, Aktan Ikiz Z. Branching patterns of the popliteal artery and its clinical importance. Surg Radiol Anat 2009; 31: 357-362

[10] Hislop M, Kennedy D, Dhupelia S. Functional popliteal artery entrapment syndrome: a review of the anatomy and pathophysiology. J Sports Med Doping Stud 2014; 4: 140

[11] Rich N, Collins G, McDonald P et al. Popliteal vascular entrapment. Its increasing interest. Arch Surg 1979; 114: 1377-1384

[12] Pillai J. A current interpretation of popliteal vascular entrapment. J Vasc Surg 2008; 48: 61S-65S

[13] Levien L, Veller M. Popliteal artery entrapment syndrome: More common than previously recognized. J Vasc Surg 1999; 30: 587-598

[14] Turnipseed W, Pozniak M. Popliteal entrapment as a result of neurovascular compression by the soleus and plantaris muscles. J Vasc Surg 1992; 15: 285-294

[15] Pillai J, Levien L, Haagensen $M$ et al. Assessment of the medial head of the gastrocnemius muscle in functional compression of the popliteal artery. J Vasc Surg 2008; 48: 1189-1196

[16] Turnipseed W. Functional popliteal artery entrapment syndrome: a poorly understood and often missed diagnosis that is frequently mistreated. J Vasc Surg 2009; 49: 1189-1195

[17] Hislop M, Kennedy D, Cramp B et al. Functional popliteal artery entrapment syndrome: poorly understood and frequently missed? A review of clinical features, appropriate investigations, and treatment options. J Sports Med (Hindawi Publ Corp) 2014; 2014: 105953

[18] Corneloup L, Labanere C, Chevalier L et al. Presentation, diagnosis, and management of popliteal artery entrapment syndrome: 11 years of experience with 61 legs. Scand J Med Sci Sports 2018; 28: 517-523

[19] Turnipseed W. Clinical review of patients treated for atypical claudication: a 28-year experience. J Vasc Surg 2004; 40: 79-85

[20] Dyer K, Hogrefe C. Don't just blame it on the veins: an update on vascular exertional limb pain. Curr Sports Med Rep 2018; 17: 347-353

[21] Hameed M, Coupland A, Davies A. Popliteal artery entrapment syndrome: an approach to diagnosis and management. $\mathrm{Br}$ J Sports Med 2018; 52: 1073-1074

[22] Chernoff D, Walker A, Khorasani R et al. Asymptomatic functional popliteal artery entrapment: Demonstration at MR imaging. Radiology 1995; 195: 176-180

[23] Lane R, Nguyen T, Cuzzilla M et al. Functional popliteal entrapment syndrome in the sportsperson. Eur J Vasc Endovasc Surg 2012; 43: $81-87$

[24] Liu Y, Sun Y, He X et al. Imaging diagnosis and surgical treatment of popliteal artery entrapment syndrome: A single-center experience. Ann Vasc Surg 2014; 28: 330-337

[25] INSITE Collaborators. Diagnosis and management of iliac artery endofibrosis: results of a delphi consensus study. Eur J Vasc Endovasc Surg 2016; 52: 90-98

[26] Veraldi G, Macrì M, Criscenti P et al. Arterial endofibrosis in professional cyclists. G Chir 2015; 36: 267-271

[27] Schep G, Bender M, van de Tempel G et al. Detection and treatment of claudication due to functional iliac obstruction in top endurance athletes: a prospective study. Lancet 2002; 359: 466-473

[28] Menon D, Onida S, Davies A. Overview of arterial pathology related to repetitive trauma in athletes. J Vasc Surg 2019; 70: 641-650 
[29] Porr ], Chrobak K, Muir B. Entrapment of the saphenous nerve at the adductor canal affecting the infrapatellar branch - a report on two cases. J Can Chiropr Assoc 2013; 57: 341-349

[30] Vento V, Faggioli G, Ancetti S et al. Cystic adventitial disease of the popliteal artery: Radical surgical treatment after several failed approaches. A case report and review of the literature. Ann Vasc Surg 2020; 64: 411.e5-411.e11

[31] Perlowski A, Jaff M. Vascular disorders in athletes. Vasc Med 2010; 15: 469-479

[32] Cassar K, Engeset J. Cystic adventitial disease: A trap for the unwary. Eur J Vasc Endovasc Surg 2005; 29: 93-96

[33] Gaunder C, McKinney B, Rivera J. Popliteal artery entrapment or chronic exertional compartment syndrome? Case Rep Med 2017; 2017: 6981047

[34] Deshpande A, Denton M. Functional popliteal entrapment syndrome. Aust N Z J Surg 1998; 68: 660-663

[35] Lohrer H, Malliaropoulos N, Korakakis V et al. Exercise-induced leg pain in athletes: diagnostic, assessment, and management strategies. Phys Sportsmed 2019; 47: 47-59

[36] Buerba R, Fretes N, Devana S et al. Chronic exertional compartment syndrome: current management strategies. Open Access J Sports Med 2019; 10: 71-79

[37] Vogels S, Ritchie E, van Dongen T et al. Systematic review of outcome parameters following treatment of chronic exertional compartment syndrome in the lower leg. Scand J Med Sci Sports 2020; 30: 1827-1845

[38] Williams E, Rosson G, Hagan R et al. Soleal sling syndrome (proximal tibial nerve compression): Results of surgical decompression. Plast Reconstr Surg 2012; 129: 454-462

[39] Pomeroy G, Wilton J, Anthony S. Entrapment neuropathy about the foot and ankle: an update. J Am Acad Orthop Surg 2015; 23: 58-66

[40] Ladak A, Spinner R, Amrami K et al. MRI findings in patients with tibial nerve compression near the knee. Skeletal Radiol 2013; 42: 553-559

[41] Chhabra A, Williams E, Subhawong T et al. MR neurography findings of soleal sling entrapment. Am J Roentgenol 2011; 196: W290-W297

[42] Burrus M, Werner B, Starman J et al. Chronic leg pain in athletes. Am J Sports Med 2015; 43: 1538-1547

[43] Gamba C, Serrano-Chinchilla P, Ares-Vidal J et al. Proximal medial gastrocnemius release versus open plantar fasciotomy for the surgical treatment in recalcitrant plantar fasciitis. Foot Ankle Int 2020; 41: 267-274

[44] Latt L, Jaffe D, Tang Y et al. Evaluation and treatment of chronic plantar fasciitis. Foot \& Ankle Orthopaedics 2020; 5: 2473011419896763

[45] Kurosawa D, Murakami E, Aizawa T. Referred pain location depends on the affected section of the sacroiliac joint. Eur Spine J 2015; 24: 521-527

[46] Smart K, Blake C, Staines A et al. Mechanisms-based classifications of musculoskeletal pain: part 2 of 3: Symptoms and signs of peripheral neuropathic pain in patients with low back ( \pm leg) pain. Man Ther 2012; 17: 345-351

[47] Vulfsons S, Bar N, Eisenberg E. Back pain with leg pain. Curr Pain Headache Rep 2017; 21: 32

[48] Shahi N, Arosemena M, Kwon J et al. Functional popliteal artery entrapment syndrome: a review of diagnosis and management. Ann Vasc Surg 2019; 59: 259-267
[49] Sirico F, Palermi S, Gambardella F et al. Ankle brachial index in different types of popliteal artery entrapment syndrome: A systematic review of case reports. J Clin Med 2019; 8: 2071

[50] Brown C, Muniz M, Kauvar D. Response of the popliteal artery to treadmill exercise and stress positioning in patients with and without exertional lower extremity symptoms. J Vasc Surg 2019; 69: 1545-1551

[51] Eliahou R, Sosna J, Bloom A. Between a rock and a hard place: clinical and imaging features of vascular compression syndromes. Radiographics 2012; 32: E33-E49

[52] Lavingia K, Dua A, Rothenberg K et al. Surgical management of functional popliteal entrapment syndrome in athletes. J Vasc Surg 2019; 70: 1555-1562

[53] Jarraya M, Simmons S, Farber A et al. Uncommon diseases of the popliteal artery: a pictorial review. Insights Imaging 2016; 7: 679-688

[54] Leiner T, Kessels A, Nelemans P et al. Peripheral arterial disease: comparison of color duplex US and contrast-enhanced MR angiography for diagnosis. Radiology 2005; 235: 699-708

[55] Elias D, White L, Rubenstein J et al. Clinical evaluation and MR imaging features of popliteal artery entrapment and cystic adventitial disease. Am J Roentgenol 2003; 180: 627-632

[56] Williams C, Kennedy D, Bastian-Jordan M et al. A new diagnostic approach to popliteal artery entrapment syndrome. J Med Radiat Sci 2015; 62: 226-229

[57] Ozkan U, Oğuzkurt L, Tercan F et al. MRI and DSA findings in popliteal artery entrapment syndrome. Diagn Interv Radiol 2008; 14: 106-110

[58] Thakor A, Chung J, Patel P et al. Use of blood pool agents with steady-state MRI to assess the vascular system. J Magn Reson Imaging 2017; 45: 1559-1572

[59] Boniakowski A, Davis F, Campbell D et al. Intravascular ultrasound as a novel tool for the diagnosis and targeted treatment of functional popliteal artery entrapment syndrome. J Vasc Surg Cases Innov Tech 2017; 3: 74-78

[60] Chou H, Wu I, Yeh K et al. The usefulness of intravascular ultrasound in popliteal artery entrapment syndrome. JACC Cardiovasc Interv 2019; 12: $2110-2111$

[61] Isner-Horobeti M, Muff G, Masat J et al. Botulinum toxin as a treatment for functional popliteal artery entrapment syndrome. Med Sci Sports Exerc 2015; 47: 1124-1127

[62] Hislop M, Brideaux A, Dhupelia S. Functional popliteal artery entrapment syndrome: use of ultrasound guided Botox injection as a non-surgical treatment option. Skeletal Radiol 2017; 46: 1241-1248

[63] Vilás R, Rodriguez L, Campos M et al. Exercise-related bilateral leg atypical claudication in female olympic taekwondo player: a case report. J Sports Sci Med 2011; 10: 768-770

[64] Kim S, Min S, Ahn S et al. Long-term outcomes after revascularization for advanced popliteal artery entrapment syndrome with segmental arterial occlusion. J Vasc Surg 2012; 55: 90-97

[65] Lejay A, Delay C, Georg Y et al. Five year outcomes of surgical treatment for popliteal artery entrapment syndrome. Eur J Vasc Endovasc Surg 2016; 51: 557-564

[66] Harriss DJ, Macsween A, Atkinson G. Ethical standards in sport and exercise science research: 2020 update. Int J Sports Med 2019; 40: 813-817 